33. Fortbildungstage der Süddeutschen Gesellschaft für Pneumologie

11.-12. Oktober 2019

Ulm, Deutschland

www.sdgp.de

Spektrum der klinischen Pneumologie

11.-12. Oktober 2019

Altötting, Deutschland

www.spektrumpneumologie-aoe.de

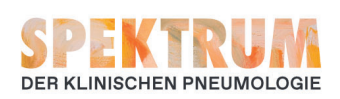

Jahrestagung der Deutschen, Österreichischen und Schweizerischen Gesellschaften für Hämatologie und Medizinische Onkologie 11.-14. Oktober 2019 Berlin, Deutschland www.haematologie-onkologie-2019.com
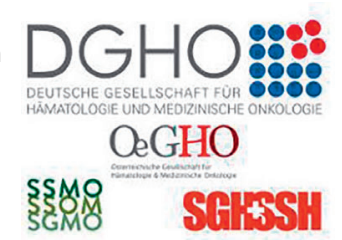

27. Jahrestagung der Deutschen Gesellschaft für Schlafforschung und Schlafmedizin (DGSM) 7.-9. November 2019 Hamburg, Deutschland www.dgsm-kongress.de

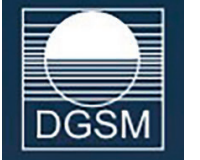

Operation Karriere

9. November 2019

Köln, Deutschland

OPERATION

30. November 2019

Berlin, Deutschland

1. Februar 2020

Frankfurt, Deutschland

www.operation-karriere.de

34. Deutscher Krebskongress

19.-22. Februar 2020

Berlin, Deutschland

www.dkk2020.de

KARRIERE

61. Kongress der Deutschen Gesellschaft für Pneumologie (DGP) Pneumologie - persönlich und präzise

25.-28. März 2020

Leipzig, Deutschland

https://pneumologie-kongress.de/

Deutsche Gesellschaft für Pneumologie und Beatmungsmedizin $\mathrm{e} V$.
11. Jahrestagung
der Atmungstherapeuten $\triangle D C P$ 12-13. ONtober 2019
Handelskammer Hamburg
34. DEUTSCHER

KREBSKONGRESS 2020

\author{
Humor auf Rezept? \\ Die Dosis bringt den Erfolg! \\ 18.-19. Oktober 2019 \\ Leipzig, Deutschland \\ www.arztmithumor.de/
}

http://jt-atmungstherapeuten-dgp.de/

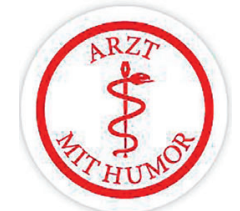

28. Jahreskongress der DIGAB e.V. und

15. Beatmungssymposium der DGP e.V.

11.-13. Juni 2020

Hamburg, Deutschland

https://digab-kongresse.de/

\section{OKTOBER 2019}

21. Herbsttagung der Mitteldeutschen Gesellschaft für Pneumologie und Thoraxchirurgie

25.-26. Oktober 2019

Dresden, Deutschland

www.mdgp.de

\section{NOVEMBER 2019}

\section{Allergiesymposium der} Norddeutschen Arbeitsgemeinschaft Pädiatrische Pneumologie und Allergologie e.V. (NAPPA)

29.-30. November 2019

Sylt/Westerland, Deutschland

www.nappa-ev.de Oktober 2019

\section{DEZEMBER 2019}

12. Deutsches Infektiologie-Update 29.-30. November 2019

6.-8. Dezember 2019

www.ifi-medizin.de

\section{FEBRUAR 2020}

8th European Conference on Tobacco or Health - ECToH 2020

19.-22. Februar 2020

Berlin, Deutschland

www.ng-akademie.de/details/ECToH2020.html

\section{MÄRZ 2020}

DACH 2020 - Pneumologische

Rehabilitation

13.-14. März 2020

Aarau, Schweiz

www.ogp.at/event/dach-2020-

pneumologische-rehabilitation/

\title{
KARGER
}

(c) 2019 S. Karger GmbH, Freiburg 